\title{
Sensitive Constrained Optimal PMU Allocation with Complete Observability for State Estimation Solution
}

\author{
M. Ravindra \\ Electrical and Electronics Engineering Department \\ Jawaharlal Nehru Technological University, Kakinada \\ Kakinada, India \\ ravieeejntu@gmail.com
}

\author{
R. Srinivasa Rao \\ Electrical and Electronics Engineering Department \\ Jawaharlal Nehru Technological University, Kakinada \\ Kakinada, India \\ srinivas.jntueee@gmail.com
}

\begin{abstract}
In this paper, a sensitive constrained integer linear programming approach is formulated for the optimal allocation of Phasor Measurement Units (PMUs) in a power system network to obtain state estimation. In this approach, sensitive buses along with zero injection buses (ZIB) are considered for optimal allocation of PMUs in the network to generate state estimation solutions. Sensitive buses are evolved from the mean of bus voltages subjected to increase of load consistently up to $50 \%$. Sensitive buses are ranked in order to place PMUs. Sensitive constrained optimal PMU allocation in case of single line and no line contingency are considered in observability analysis to ensure protection and control of power system from abnormal conditions. Modeling of ZIB constraints is included to minimize the number of PMU network allocations. This paper presents optimal allocation of PMU at sensitive buses with zero injection modeling, considering cost criteria and redundancy to increase the accuracy of state estimation solution without losing observability of the whole system. Simulations are carried out on IEEE 14, 30 and 57 bus systems and results obtained are compared with traditional and other state estimation methods available in the literature, to demonstrate the effectiveness of the proposed method.
\end{abstract}

Keywords—sensitive; bus; observability; phasor; measurement; pmu; state; estimation; zero; injection; zib

\section{INTRODUCTION}

State estimation plays a vital role in real-time control of power system providing security and reliability. It acts as a filter between the received information and application functions that need reliable data. In power systems, supervisory control and data acquisition (SCADA) systems are used to collect the raw data (bus voltage magnitudes, currents and complex power flows) of the transmission network and this data is processed for state estimation solution. However, this information is not sufficient to estimate the accurate states of voltage and phase angles at every bus in the system. One of the recent developments in real time analysis of power systems is an implementation of synchrophasor in the state estimation process. Synchrophasors or PMUs are used to directly measure phase angles associated with voltages and currents with respect to an absolute time reference. This absolute reference is provided by common timing signal by high accuracy clocks to Coordinated Universal Time (UTC) such as Global Positioning
Systems (GPS) [1]. A phasor measuring unit is a device used to store synchronized measurement data and to communicate the same to a control center through GPS. Allocation of PMUs at every bus to measure the complete data of the system is not a feasible solution from economic perspective. Hence, in order to get complete observability and identify gross errors in measurement set, PMUs must be placed optimally at sensitive nodes by considering ZIB in the network. In a power system network, some of the buses are sensitive to sudden load changes that may lead to a blackout. Blackouts occur in power grid predominantly due to inadequate generation and state predictability of the system [2]. Prior knowledge of the states of a system at sensitive buses can be very useful in avoiding blackouts. Different types of sensitive indices are proposed in the literature for various applications in the performance analysis of power system [3-7]. In this paper voltage sensitive indices are used to identify sensitive buses for the placement of PMUs.

Several approaches are proposed in literature for optimal PMU placement. Initial work on phasor measurements using Synchrophasors to measure phasor voltage and currents is proposed in [8]. Authors in [9] proposed a Linear Programming based measurement system for maintaining observability when a single line outage occurs in the network. Authors in [10] used GA with the immune operator to select optimal sites for PMU placement to achieve observability with improved converging speed and execution time. Authors in [11] implemented Nondominated Sorting Genetic Algorithm (NSGA) in PMU placement problem and simultaneously optimized two conflicting objectives such as minimization of number of PMUs and maximization of the measurement redundancy and obtained Pareto-optimal solutions. Authors in [12] developed an integrated model to show the effect of ZIB and conventional measurements in PMU allocation to enhance system observability by considering single branch and single PMU outage contingencies separately and simultaneously. Authors in [13] proposed a method for multi-staging of PMUs by modeling zero injection constraint as a linear model in integer linear programming approach with two indices such as Bus Observability Index (BOI) and System Observability Redundancy Index (SORI). Author in [14] proposed a generalized linear integer programming approach for redundant 
PMU placement, full and incomplete observability, with and without zero injections and showed that accuracy, redundancy, and robustness of state estimation solution will be enhanced with PMU placement. Authors in [15] considered the PMU placement problem as a quadratic minimization problem with continuous decision variables and solved the problem using non-linear weighted least squares approach. Authors in [16] proposed a binary search algorithm to determine the minimum number of PMUs for observability under normal conditions and single branch outage conditions. Authors in [17] introduced the concept of time-synchronized measurements for PMU placement and solved the problem using integer quadratic programming to minimize the total number of PMUs and to increase the redundancy at power system buses. In [18], authors formulated a binary integer linear programming approach with binary decision variables to locate PMUs at each bus by integrating possibility of single or multiple PMU loss in the decision strategy while preserving observability and the lowest metering economy. Authors in [19] introduced a hybrid constrained state estimator in which conventional and synchrophasor measurements are incorporated simultaneously in the problem without using any measurement transformation and shown that solution converges faster with small uncertainty. Authors in [20] introduced a multistage state estimation procedure to include synchrophasor measurements without disturbing existing SCADA system. This procedure requires more PMUs which opposes the economic criteria. Authors in [21] investigated three different methods to include PMU measurements into state estimation problem.

In this paper, a sensitive constrained integer linear programming approach is used for optimal PMU allocation considering sensitive buses along with ZIBs. The PMUs are placed at sensitive buses which are identified based on voltage stability index with complete observability. The method is tested with different test cases and results are presented. The rest of the paper is organized as follows: Section II deals with traditional state estimation method; Section III covers optimal PMU allocation considering sensitive buses; Section IV presents state estimation with conventional and PMU measurements. Section $\mathrm{V}$ provides results and analysis and Section VI gives conclusions.

\section{TRADITIONAL STATE ESTIMATION}

The traditional state estimation method collects data from SCADA systems to find states of power systems. For a given set of measurements, weighted least squares state estimation [22] can be represented as:

$$
\operatorname{Min} \sum_{\mathrm{k}=1}^{\mathrm{m}} \mathrm{W}_{\mathrm{kk}} r_{k}^{2}
$$

subjected to: $\quad Z_{k}=h_{k}(x)+r_{k}, \mathrm{k}=1 \ldots \mathrm{m}$

where $r_{k}=Z_{k}-h_{k}(x)$ is residual value of measurement $k$ calculated from the difference of the measured $Z_{k}$ and a nonlinear function $h_{k}(x)$ related to measurement state vector (x).
The residual vector $r_{k}^{2}$ is weighted by $W_{k k}=\sigma_{k}^{-2}$ which is calculated from the standard deviation of respective measurements and $\mathrm{m}$ is the number of measurements. The gain matrix is formed for better accurate state estimation, formulated with Jacobian $\mathrm{H}$ matrix and covariance $R=\operatorname{diag}\left\{\sigma_{1}^{2}, \sigma_{2}^{2} \ldots \ldots \sigma_{m}^{2}\right\}$. The Jacobian matrix for traditional state estimation is defined as

$$
H=\left[\begin{array}{cc}
\frac{\partial P_{i n}}{\partial \delta} & \frac{\partial P_{i n}}{\partial v} \\
\frac{\partial Q_{i n}}{\partial \delta} & \frac{\partial Q_{i n}}{\partial v} \\
\frac{\partial P_{f l}}{\partial \delta} & \frac{\partial P_{f l}}{\partial v} \\
\frac{\partial Q_{f l}}{\partial \delta} & \frac{\partial Q_{f l}}{\partial v} \\
0 & \frac{\partial v_{m a g}}{\partial v}
\end{array}\right]
$$

The gain matrix is defined as

$$
G(\Delta x)=H^{T} R^{-1} \Delta Z
$$

where $G=H^{T} R^{-1} H$ and $\Delta Z=Z-h_{k}(x)$

From (4), the solution is obtained by updating the state vector

$$
x^{t+1}=x^{t}+\Delta x
$$

The convergence of the state estimation algorithm is obtained when $\Delta \mathrm{x}$ becomes smaller than the tolerance value $10^{-}$ 5 . In state estimation process observability of the system can be obtained numerically by finding the rank of the Jacobian matrix or through topological bus connectivity matrix. If Jacobian matrix $\mathrm{H}$ has full rank the system is numerically observable otherwise is not observable. In this paper topological observability is considered, each bus is checked with Redundancy Index (RI) and total system with Complete System Observability Redundancy Index (CSORI) to show complete observability of the system.

\section{OPTIMAL PMU ALLOCATION CONSIDERING SENSITIVE BUSES}

\section{A. Formulation for the Optimal PMU Allocation Problem}

The proposed approach is formulated as an optimization problem of allocating PMUs in the network with highest preference at sensitive buses for complete observability considering cost criteria which produce accurate state estimation solution. The optimization problem for optimal allocation of PMUs is formulated as:

$$
\operatorname{Min} \sum_{\mathrm{k}=1}^{\mathrm{n}} \mathrm{F}_{\mathrm{k}} x_{k}
$$

Subject to $\quad A X \geq B$ and $A_{e q} X=B_{e q}$ 
where $F_{k}$ is cost coefficient of PMU installed at bus k, $A_{e q}$ is matrix of order $n \times n$ consisting of sensitive buses, whose entries are all equal to one and for other buses it is zero, $\mathrm{n}$ is number of buses, $X=\left[\begin{array}{lllll}x_{1} & x_{2} & x_{3} & \ldots & x_{n}\end{array}\right]^{T}$ is a decision variable vector, $\mathrm{B}$ and $\mathrm{B}_{\mathrm{eq}}$ is observability constraints matrix which can be written as $\left[\begin{array}{lllll}1 & 1 & 1 & 1 & \ldots . .1\end{array}\right]_{n \times 1}^{T}, x_{k}$ is binary decision variable and $A$ is incidence matrix of order $i \times j$ which are defined as

$$
\begin{aligned}
& x_{k}=\left\{\begin{array}{lr}
1 & \text { if PMU is installed at bus } k \\
0 & \text { otherwise }
\end{array}\right. \\
& A_{i, j}=\left\{\begin{array}{cr}
1 & \text { if } i=j \text { or connected to each other } \\
0 & \text { otherwise }
\end{array}\right.
\end{aligned}
$$

Proper identification of sensitive buses in the system and optimal placement of PMUs at these buses accurately estimate the states of state estimation problem.

\section{B. Identification of Sensitive Buses}

From experiences of major blackouts occurred in India, failure of the supply occurred due to either sudden removal of generation or overloading beyond safety limits leading to dip in system voltage. In order to determine the most sensitive buses in a system which are prone to load changes, voltage stability indexes (VSI) at each bus are formulated by increasing the load from 5 to $50 \%$.

$$
\begin{aligned}
& V_{\text {avg }}=\frac{1}{N} \sum_{i=1}^{N} V(i) \\
& V S I=V_{a v g}-V_{0}
\end{aligned}
$$

where, $\mathrm{V}_{0}$ is the vector of true bus voltages obtained from the base load, $V_{\text {avg }}$ is calculated from average of voltages obtained with increased load and $\mathrm{N}$ is the number of load samples. VSI is calculated from the difference of average voltage and true bus voltage. The bus with the highest VSI value among buses is considered as the highest sensitive bus in the system. The calculated values of VSI for 14-bus test case system are shown in Table I. From Table I data, sensitive buses are arranged in descending order i.e., $\mathrm{B} 14>\mathrm{B} 9>\mathrm{B} 10>\mathrm{B} 4>\mathrm{B} 7>\mathrm{B} 5>\mathrm{B} 11$ $>\mathrm{B} 13>\mathrm{B} 12$, where $\mathrm{B} 1$ is slack bus $\mathrm{B} 2, \mathrm{~B} 3, \mathrm{~B} 6$, and $\mathrm{B} 8$ are generator buses, B7 is ZIB and remaining are load buses. B14 and B9 buses are the most sensitive buses (in that order) affected by load change. In this paper, sensitive buses are considered as constraints for optimal PMU allocation. The procedure to compute voltage stability index (VSI) and sensitive buses at each bus is presented in Figure 1.

TABLE I. VSI DATA OBTAINED FROM LOAD FLOWS

\begin{tabular}{|c|c|c|c|c|c|c|c|}
\hline Bus no & B1 & B2 & B3 & B4 & B5 & B6 & B7 \\
\hline VSI & 0 & 0 & 0 & 0.0062 & 0.0059 & 0 & 0.0062 \\
\hline Bus no & B8 & B9 & B10 & B11 & B12 & B13 & B14 \\
\hline VSI & 0 & 0.0095 & 0.0094 & 0.0055 & 0.0036 & 0.0051 & 0.0115 \\
\hline
\end{tabular}

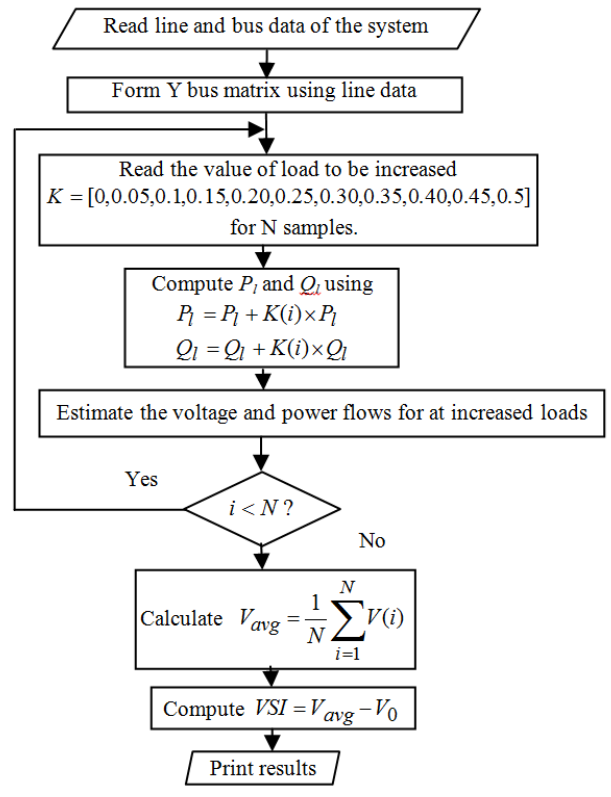

Fig. 1. Flowchart to estimate sensitive buses

\section{Sensitive Constrained Optimal PMU Allocation}

Placement of PMU at each and every bus increases the number of PMUs in the system which is a drawback from economic perspective. Hence, in order to optimize the number of PMUs and to allocate PMUs at sensitive buses for accurate state estimation solution, the problem is formulated as follows using an eight-bus network as shown in Figure 2.

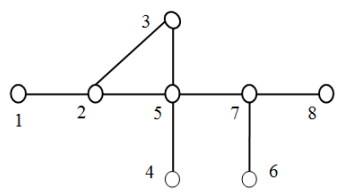

Fig. 2. Eight- bus system network diagram

The optimization problem can be expressed as

$\operatorname{Min} x_{1}+x_{2}+x_{3}+\ldots \ldots \ldots x_{8}$

Subject to observability constraints

Bus 1: $\quad x_{1}+x_{2} \geq 1$

Bus 2:

$$
x_{1}+x_{2}+x_{3}+x_{5} \geq 1
$$

Bus 3:

$$
x_{2}+x_{5}+x_{3} \geq 1
$$

Bus 4:

$$
x_{4}+x_{5} \geq 1
$$

Bus 5:

$$
x_{2}+x_{3}+x_{4}+x_{5} \geq 1
$$

Bus 6:

$$
x_{6}+x_{7} \geq 1
$$

Bus 7:

$$
x_{5}+x_{6}+x_{7}+x_{8} \geq 1
$$

Bus 8:

$$
x_{7}+x_{8} \geq 1
$$


where $x_{k} \in\{0,1\}$ in sensitive constrained integer linear programming formulation. For instance, consider bus 5 and 7 are the most sensitive buses in the eight-bus system. In order to allocate PMU at sensitive buses, locations suggested are $x_{5}=1, x_{7}=1$. As a result of substituting these values in observability constraints (11-18), inequalities for buses 2, 3, 4, $5,6,7,8$ are satisfied. After eliminating satisfied constraints, residual observable constraints subjected to optimization are shown below. The sensitive constrained optimization problem is written as

$$
\text { Minimize } \sum_{k=1}^{8} x_{k}
$$

Subjected to observability constraints $x_{1}+x_{2} \geq 1$

With application of integer linear programming the solution $\left[\begin{array}{llllllll}0 & 1 & 0 & 0 & 1 & 0 & 1 & 0\end{array}\right]$ which means PMUs need to be allocated at 2, 5, 7 to make the system completely observable.

\section{Optimization of Sensitive Constrained PMUs Allocation with Zero Injection (ZI) Modeling}

In a power system when there is no generation or load at any particular bus, power injection to rest of the network is zero. Such buses are considered as ZIBs. When ZIBs are considered, the size of connectivity matrix will be reduced, thus it is possible to minimize the number of PMUs in the network. In this problem of zero injection modeling, every bus connected to ZIB is considered. Permutation matrix $\mathrm{P}$ is formulated from an array of the vector that includes buses not associated with ZIB and array of buses associated with ZIB. ZIB constraint matrix is formulated with the number of buses not associated with ZIB and buses associated with ZIB. The buses associated with only ZIB can be written as $Z_{\text {as }}$.

ZIB constraint matrix is written as

$$
Z_{M}=\left[\begin{array}{cc}
I_{L \times L} & 0 \\
0 & Z_{a s}
\end{array}\right]
$$

where $\mathrm{I}_{\mathrm{L} \times \mathrm{L}}$ is identity matrix of $\mathrm{L}$ number of buses not associated with ZIB. The bus connectivity matrix developed for optimal allocation of PMU can be presented as

$$
Z_{p m u}=A^{*} P^{*} Z_{M}
$$

where, A is bus incidence matrix defined from the bus system, $P$ is permutation matrix of the bus system and $Z_{m}$ is $Z I B$ constraint matrix formulated in (21). Bus constraint matrix $b_{\text {con }}$ is defined as the vector of buses (not associated with ZIB which are all equal to one) and the number of buses associated with each ZIB. Bus constraint matrix is vector formed to check observability of the system. The optimization of sensitive constrained PMUs with zero injection modeling is formulated as

$$
\operatorname{Min} \sum_{\mathrm{k}=1}^{\mathrm{n}} \mathrm{F}_{\mathrm{k}} x_{k}
$$

subjected to observability constraints

$$
\begin{aligned}
& Z_{\text {pmu }} X \geq b_{\text {con }} \\
& A_{e q} X=B_{e q}
\end{aligned}
$$

where: $X=\left[x_{1} x_{2} \ldots \ldots x_{N}\right]^{T}, x_{k} \in(0,1), k=1,2,3, \ldots, n, Z_{p m u}$ is bus connectivity matrix defined in (22), $b_{\text {con }}$ and $B_{\text {eq }}$ is bus constraint matrix formed to check observability of the system. $\mathrm{A}_{\mathrm{eq}}$ is a matrix of $\mathrm{n} \times \mathrm{n}$ order which consists of sensitive bus elements whose entries are equal to one and for other bus elements it is zero. Equation (25) ensures that $\mathrm{x}_{\mathrm{k}}$ variable must be one for sensitive buses in the system. It gives priority to sensitive buses for allocation of PMUs in the bus network. For instance, in Figure 2, bus-2 is a ZIB which is optimized with modeling of bus connectivity matrix. Permutation matrix of the system is

$$
P=\left[\begin{array}{llllllll}
0 & 0 & 0 & 1 & 0 & 0 & 0 & 0 \\
0 & 0 & 0 & 0 & 0 & 1 & 0 & 0 \\
0 & 0 & 0 & 0 & 0 & 0 & 1 & 0 \\
0 & 0 & 0 & 0 & 0 & 0 & 0 & 1 \\
1 & 0 & 0 & 0 & 0 & 0 & 0 & 0 \\
0 & 1 & 0 & 0 & 0 & 0 & 0 & 0 \\
0 & 0 & 1 & 0 & 0 & 0 & 0 & 0 \\
0 & 0 & 0 & 0 & 1 & 0 & 0 & 0
\end{array}\right]
$$

From (21) ZIB constraint matrix is written as

$$
Z_{m}=\left[\begin{array}{llllllll}
1 & 0 & 0 & 0 & 0 & 0 & 0 & 0 \\
0 & 1 & 0 & 0 & 0 & 0 & 0 & 0 \\
0 & 0 & 1 & 0 & 0 & 0 & 0 & 0 \\
0 & 0 & 0 & 1 & 0 & 0 & 0 & 0 \\
0 & 0 & 0 & 0 & 1 & 1 & 1 & 1
\end{array}\right]
$$

Bus constraint matrix is developed such that every bus of the system is measured by at least two PMUs.

$b_{\text {con }}=\left[\begin{array}{lllll}1 & 1 & 1 & 1 & 3\end{array}\right]^{T}$

From (22), bus connectivity matrix for optimal allocation of PMUs can be calculated as

$$
Z_{p т u}=\left[\begin{array}{llllllll}
0 & 0 & 0 & 1 & 1 & 0 & 0 & 0 \\
0 & 0 & 0 & 0 & 0 & 1 & 1 & 0 \\
0 & 0 & 0 & 0 & 1 & 1 & 1 & 1 \\
0 & 0 & 0 & 0 & 1 & 1 & 1 & 1 \\
2 & 4 & 3 & 1 & 3 & 0 & 1 & 0
\end{array}\right]
$$

$B_{\text {con }}, A_{\text {eq }}, F, Z_{\text {pmu }}$ and number of non- zero elements in $A_{\text {eq }}$ are inputs for sensitive constrained integer linear programming

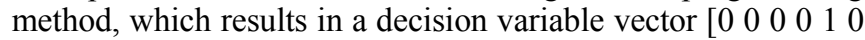
$10]$. This gives optimal allocation of PMUs at 5 and 7 buses.

\section{E. Optimization of Sensitive Constrained PMUs Allocation under Single Line Contingency}

The optimization of sensitive constrained PMUs with zero injection modeling in case single line contingency is formulated as 


$$
\operatorname{Min} \sum_{\mathrm{k}=1}^{\mathrm{n}} \mathrm{F}_{\mathrm{k}} x_{k}
$$

subjected to observability constraints

$$
\begin{gathered}
Z_{p m u} X \geq 2 b_{c o n} \\
A_{e q} X=B_{e q}
\end{gathered}
$$

In case of single line contingency, the problem is formulated in such a way that each network bus is observed by at least two PMUs

\section{F. Complete Observability of the System}

The performance of optimization method depends on the observability of the power system. The performance of every bus of the system is measured by Bus Observability Index (BOI). The maximum BOI is limited to maximum connectivity $(\chi)$ of the bus plus one [13].

$$
\mathfrak{J}_{k} \leq \chi_{k}+1
$$

For bus-k, ( $\mathfrak{J}$ ) BOI gives the number of times the bus is observed by the PMU. To know the performance of the total system, Complete System Observability Redundancy Index (CSORI) is determined and it can be presented as the sum of BOI of every bus in the system.

$$
\text { CSORI }=\sum_{k=1}^{n} \mathfrak{I}_{k}
$$

Maximum redundancy of the bus can be formulated as

$$
\operatorname{Max} \sum_{k=1}^{n} b_{k}{ }^{T} Z_{p m u} x_{k}
$$

subjected to following constraints

$$
\begin{aligned}
& \sum_{k=1}^{n} x_{k}=\mu_{0} \\
& Z_{p m u} X \geq b
\end{aligned}
$$

where $\mu 0$ is the minimum number of PMUs obtained for complete system observability, Zpmu is bus connectivity matrix obtained from the proposed sensitive constrained approach. $b$ is the vector of observability constraints which is written as transpose of vector $\left[\begin{array}{llllll}1 & 1 & 1 & 1 & \ldots & \ldots\end{array}\right]$.

\section{STATE ESTIMATION WITH CONVENTIONAL AND OPTIMAL SENSITIVE CONSTRAINED PMU MEASUREMENTS}

Newton-Raphson load flow is considered for calculation of conventional measurements and true state values $\left(\mathrm{V}_{\text {tru }}, \delta_{\text {tru }}\right)$ of the system. For integration of PMU measurements into state estimation, the method followed in this paper is widely investigated in [19], [21], [25-27] and this process exhibits good results. For the joint optimality of PMU and SCADA measurements, the optimization method used for solving nonlinear equations is WLS method in which the errors are weighted with standard deviation. The proposed method includes optimal PMU measurements at sensitive buses along with ZIBs obtained from sensitive constrained ILP approach formulated in this paper. The procedure to find states of the power system with sensitive constrained state estimation method is presented in Figure 3. The measurement function for state estimation is written as

$$
Z_{k}=h_{k}(x)+r_{k}
$$

where $Z_{k}$ is a vector of conventional and optimal PMU measurements, $x$ is state vector, $h_{k}$ is the measurement vector of non-linear function related to state vector $\mathrm{x}$ and $r_{k}$ is measurement error vector. The residual vector obtained from the measurement function can be written as

$\mathrm{r}_{\mathrm{k}}=\mathrm{Z}_{\mathrm{k}}-\mathrm{h}_{\mathrm{k}}(\mathrm{x}), \mathrm{k}=1,2 \ldots \mathrm{m}$

The objective function for sensitive constrained state estimation is formulated as

$J(x)=\operatorname{Min} \sum_{k=1}^{m} \frac{\left(Z_{k}-h_{k}(x)\right)^{2}}{R_{k k}}$

$\mathrm{R}_{\mathrm{kk}}$ is diagonal matrix formed with inverse of variances of conventional and PMU measurements which is written as

$\mathrm{R}_{\mathrm{kk}}=$ diagonal of $\left[1 / \sigma_{1}^{2}, 1 / \sigma_{2}^{2}, \ldots \ldots .1 / \sigma_{m}^{2}\right]$

where $\sigma_{m}^{2}$ is covariance of the measurements. The state estimation method integrates PMU measurements with conventional measurements by designing Jacobian matrix i.e. first order derivatives of PMU and conventional measurements are formulated in the state estimation process. The Jacobian matrix with PMU measurements at sensitive buses and conventional measurements can be formulated as

$$
H_{p m u}=\left[\begin{array}{cc}
\frac{\partial P_{i n}}{\partial \delta} & \frac{\partial P_{i n}}{\partial v} \\
\frac{\partial Q_{i n}}{\partial \delta} & \frac{\partial Q_{i n}}{\partial v} \\
\frac{\partial P_{f l}}{\partial \delta} & \frac{\partial P_{f l}}{\partial v} \\
\frac{\partial Q_{f l}}{\partial \delta} & \frac{\partial Q_{f l}}{\partial v} \\
0 & \frac{\partial v_{p m u}}{\partial v} \\
\frac{\partial \theta_{p m u}}{\partial \delta} & 0 \\
\frac{\partial I_{r p m u}}{\partial \delta} & \frac{\partial I_{r p m u}}{\partial v} \\
\frac{\partial I_{i p m u}}{\partial \delta} & \frac{\partial I_{i p m u}}{\partial v}
\end{array}\right]
$$




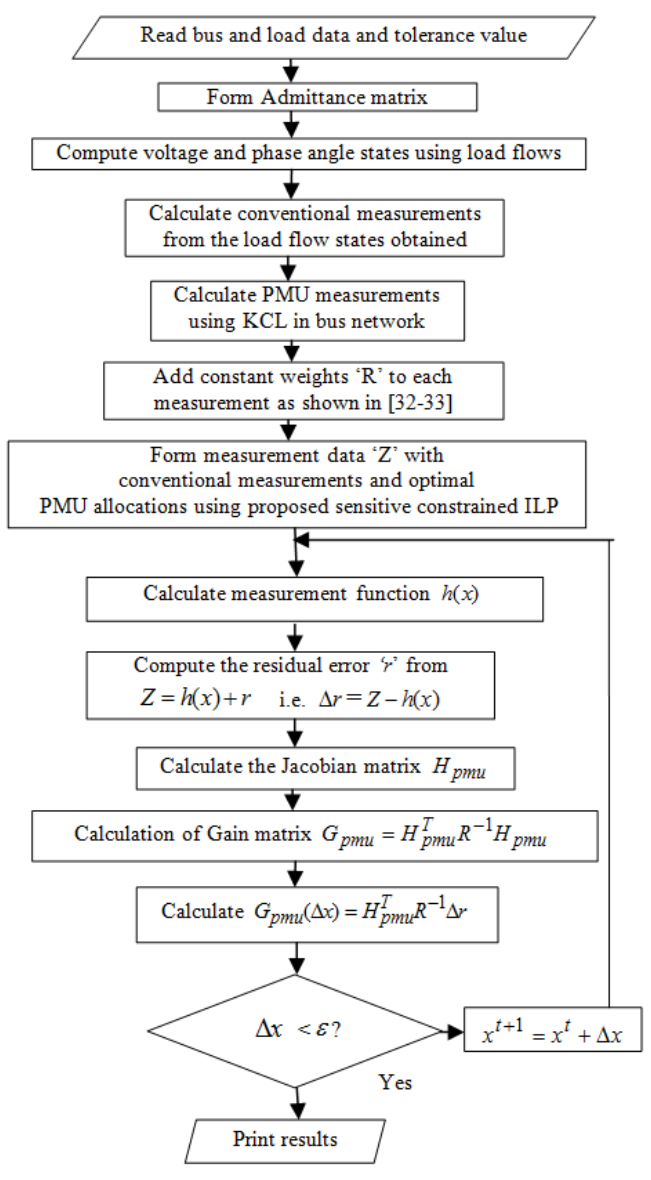

Fig. 3. Flow chart for state estimation with optimal allocation of PMUs considering sensitive buses

Gain matrix is defined for the best solution of the system state accuracy which is written as

$$
G_{p m u}(\Delta x)=H_{p m u}^{T} R^{-1} \Delta r
$$

where $G_{p m u}=H_{p m u}^{T} R^{-1} H_{p m u} \quad, \quad \Delta r=Z-h(x) \quad$. State estimation solution is obtained by updating state vector which is defined as

$$
\begin{aligned}
& \Delta x=\left(H_{p m u}^{T} R^{-1} H_{p m u}\right)^{-1} H_{p m u}^{T} R^{-1}\left(Z-h\left(x^{t}\right)\right) \\
& x^{t+1}=x^{t}+\left(H_{p m u}^{T} R^{-1} H_{p m u}\right)^{-1} H_{p m u}^{T} R^{-1}\left(Z-h\left(x^{t}\right)\right)
\end{aligned}
$$

where $x^{t}$ is the vector of state variables at $t^{\text {th }}$ iteration. The convergence tolerance $(\varepsilon)$ for state estimation is selected as $10^{-5}$ and state error is obtained from the difference of estimated states $\left(\mathrm{V}_{\text {est }}, \delta_{\text {est }}\right)$ and true values of the system. Voltage magnitude error $\left(\mathrm{V}_{\text {err }}\right)$ and phase angle error $\left(\delta_{\text {err }}\right)$ are written as

$$
V_{e r r}=V_{e s t}-V_{t r u}, \delta_{e r r}=\delta_{e s t}-\delta_{t r u}
$$

\section{RESULTS AND ANALYSIS}

In order to allocate PMUs and estimate the states of a power system, the proposed method is applied to 14, 24, 30 and 57-bus test systems. The single line diagrams of 14 and 57-bus system are shown in Figures 4 and 5 respectively. Simulations are carried out on the test systems with MATLAB and results are presented and compared. MATLAB programs are run on Intel(R) core(TM), i3 processor at $2.20 \mathrm{GHz}$ with $4 \mathrm{~GB}$ of RAM. Table II shows the zero injection buses, and sensitive buses generated from formulation (8), (9).

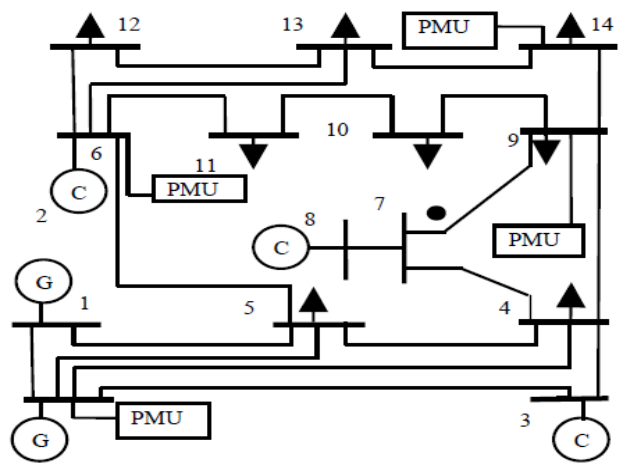

Fig. 4. Sensitive constrained PMU locations in 14 bus network

TABLE II. SENSITIVE AND ZERO INJECTION BUSES

\begin{tabular}{|c|c|c|}
\hline $\begin{array}{c}\text { IEEE test } \\
\text { systems }\end{array}$ & $\begin{array}{c}\text { Sensitive } \\
\text { buses }\end{array}$ & ZIB buses \\
\hline 14 bus & 14,9 & 7 \\
\hline 24 bus & 22,21 & $11,12,17,24$ \\
\hline 30 bus & $30,26,24,19$ & $6,9,22,25,27,28$ \\
\hline 57 bus & $31,33,29,32,25$ & $\begin{array}{c}4,7,11,21,22,24,26,34, \\
36,37,39.40,45,46,48\end{array}$ \\
\hline
\end{tabular}

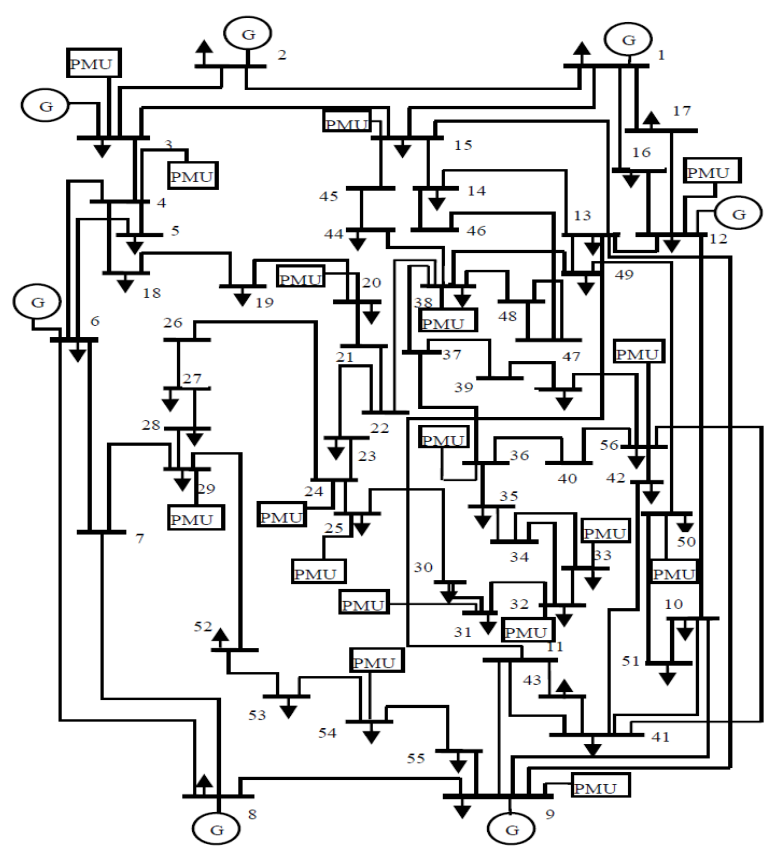

Fig. 5. Sensitive constrained PMU allocation for 57 bus system. 


\section{A. Optimal PMU Allocation with Sensitive Constrained Integer Linear Programming}

General optimal PMU locations without sensitive constraints using BILP method [30] is shown in Table III.

TABLE III. PMU ALLOCATIONS WITHOUT SENSITIVE CONSTRAINTS

\begin{tabular}{|c|c|c|}
\hline $\begin{array}{c}\text { IEEE } \\
\text { Test } \\
\text { Systems }\end{array}$ & $\begin{array}{c}\text { No. of } \\
\text { PMUs }\end{array}$ & PMU Locations \\
\hline 14bus & 4 & $2,6,7,9$ \\
\hline $\mathbf{2 4}$ bus & 7 & $2,3,8,10,16,21,23$ \\
\hline 30 bus & 10 & $1,7,9,10,12,18,24,25,27,28$ \\
\hline $\mathbf{5 7}$ bus & 17 & $1,4,6,13,19,22,25,27,29,32,36,39,41,45,47,51,54$ \\
\hline
\end{tabular}

Binary integer programming is used to model sensitive constrained ILP method for optimization. Using bus constraint vector matrix and bus connectivity matrix, zero injection modeling is framed in sensitive constrained ILP to optimize PMU allocation in the network. The sensitive buses are arranged according to the priority of higher sensitive buses. Table IV shows sensitive constrained PMU allocation with and without out zero injection modeling. Table $\mathrm{V}$ shows sensitive constrained PMU allocation with and without zero injection modeling under single line contingency. With ZIB modeling, allocation of PMUs in the network is reduced to meet the cost criteria. The method's performance is analyzed through the system redundancy. Redundancy Index (RI) measures the observability of each bus of the system covered under PMUs. System redundancy increases with the optimal location of PMUs in the system.

Tables VI and VII show the redundancy index of sensitive constrained PMU locations under no line and single line contingency. It can be observed from Table VI that under single line contingency PMUs are placed in the network in a way that each branch is observable by at least 2 PMUs. Table VIII shows the comparison of CSORI that is observable with the optimal number of PMUs under no line and single line contingency in the system.

TABLE IV. SENSITIVE CONSTRAINED PMU ALLOCATIONS WITH AND WITHOUT ZI MODELING

\begin{tabular}{|c|c|c|c|c|}
\hline \multirow{2}{*}{$\begin{array}{c}\text { IEEE } \\
\text { Test } \\
\text { systems }\end{array}$} & \multicolumn{2}{|c|}{ Without ZI Modeling } & \multicolumn{2}{|c|}{ With ZI Modeling } \\
\hline & $\begin{array}{l}\text { No. of } \\
\text { PMUs }\end{array}$ & PMU locations & $\begin{array}{l}\text { No. of } \\
\text { PMUs }\end{array}$ & PMU locations \\
\hline 14bus & 5 & $2,6,7,9,14$ & 4 & $2,6,9,14$ \\
\hline 24 bus & 8 & $3,4,8,10,16,21,22,23$ & 7 & $1,4,6,8,19,21,22$ \\
\hline 30 bus & 10 & $\begin{array}{c}3,6,7,9,10,12,19,24 \\
26,30\end{array}$ & 9 & $\begin{array}{c}3,6,7,10,12,19,24, \\
26,30\end{array}$ \\
\hline 57 bus & 20 & $\begin{array}{c}1,4,9,12,20,24,25,28 \\
29,31,32,33,36,38,39 \\
41,45,46,50,53\end{array}$ & 17 & $\begin{array}{c}3,4,9,12,15,20,24, \\
25,29,31,32,33,36, \\
38,50,54,56\end{array}$ \\
\hline
\end{tabular}

Table IX shows the number of PMUs allocated with proposed method and other methods [10, 12-17]. The proposed approach optimizes and allocates PMUs at sensitive buses that are prone to load changes. PMU allocation at sensitive buses is given the highest preference to provide accurate states of the system. All other methods cited in [10, 12-17] optimized the
PMUs without considering the constraints of the buses and allocation of PMUs at sensitive buses which results in inaccurate system measurements.

TABLE V. SENSITIVE CONSTRAINED PMU ALLOCATIONS WITH AND WITHOUT ZI MODELING UNDER SINGLE LINE CONTINGENCY

\begin{tabular}{|c|c|c|c|c|}
\hline \multirow{2}{*}{$\begin{array}{c}\text { IEEE } \\
\text { Test } \\
\text { systems }\end{array}$} & \multicolumn{2}{|c|}{ Without ZI Modeling } & \multicolumn{2}{|c|}{ With ZI Modeling } \\
\hline & $\begin{array}{l}\text { No. of } \\
\text { PMUs }\end{array}$ & PMU locations & $\begin{array}{l}\text { No. of } \\
\text { PMUs }\end{array}$ & PMU locations \\
\hline 14bus & 10 & $2,4,5,6,7,8,9,11,13,14$ & 8 & $2,4,5,6,9,10,13,14$ \\
\hline 24 bus & 15 & $\begin{array}{c}1,2,3,7,8,9,10,11,15 \\
16,17,20,21,22,23\end{array}$ & 12 & $\begin{array}{c}1,2,7,8,9,, 10,16 \\
18,19,21,22,23\end{array}$ \\
\hline 30 bus & 21 & $\begin{array}{c}1,2,3,5,6,8,9,10,11,12 \\
13,15,16,18,19,22,24,25 \\
26,27,30\end{array}$ & 17 & $\begin{array}{c}1,3,5,6,7,10,12,13 \\
15,17,19,20,22,24 \\
26,27,30\end{array}$ \\
\hline 57 bus & 33 & $\begin{array}{c}1,3,4,6,9,12,15,19,20, \\
22,24,25,27,28,29,31,32, \\
33,35,36,37,38,39,41,43 \\
45,46,47,50,51,53,54,56\end{array}$ & 30 & $\begin{array}{c}1,2,4,6,9,12,15,18 \\
20,22,24,25,28,29, \\
30,31,32,33,35,36, \\
38,41,45,48,49,50, \\
51,53,54,56\end{array}$ \\
\hline
\end{tabular}

TABLE VI. RI OF BUS WITH SENSITIVE CONSTRAINED PMU ALLOCATIONS

\begin{tabular}{|c|c|c|c|c|c|c|c|}
\hline Bus No & B1 & B2 & B3 & B4 & B5 & B6 & B7 \\
\hline RI & 1 & 1 & 1 & 3 & 2 & 1 & 2 \\
\hline Bus No & B8 & B9 & B10 & B11 & B12 & B13 & B14 \\
\hline RI & 1 & 3 & 1 & 1 & 1 & 2 & 2 \\
\hline
\end{tabular}

TABLE VII. RI OF BUS WITH SENSITIVE CONSTRAINED PMU ALLOCATIONS UNDER SINGLE LINE CONTINGENCY

\begin{tabular}{|c|c|c|c|c|c|c|c|}
\hline Bus No & B1 & B2 & B3 & B4 & B5 & B6 & B7 \\
\hline RI & 2 & 3 & 2 & 5 & 4 & 4 & 4 \\
\hline Bus No & B8 & B9 & B10 & B11 & B12 & B13 & B14 \\
\hline RI & 2 & 4 & 2 & 2 & 2 & 3 & 3 \\
\hline
\end{tabular}

TABLE VIII. CSORI FOR SENSITIVE CONSTRAINED PMU LOCATIONS

\begin{tabular}{|c|c|c|c|c|}
\hline \multirow{2}{*}{$\begin{array}{c}\text { IEEE } \\
\text { Test } \\
\text { systems }\end{array}$} & \multicolumn{2}{|c|}{ No Line Contingency } & \multicolumn{2}{c|}{ Single Line Contingency } \\
\cline { 2 - 5 } & $\begin{array}{c}\text { CSORI } \\
\text { Modh ZI } \\
\text { Moding }\end{array}$ & $\begin{array}{c}\text { CSORI } \\
\text { without } \\
\text { ZI } \\
\text { Modeling }\end{array}$ & $\begin{array}{c}\text { CSORI } \\
\text { with ZI } \\
\text { Modeling }\end{array}$ & $\begin{array}{c}\text { CSORI } \\
\text { without ZI } \\
\text { Modeling }\end{array}$ \\
\hline 14 bus & 18 & 22 & 36 & 42 \\
\hline 24 bus & 24 & 33 & 48 & 62 \\
\hline 30 bus & 39 & 43 & 67 & 82 \\
\hline 57 bus & 72 & 79 & 120 & 128 \\
\hline
\end{tabular}

TABLE IX. COMPARISON OF OPTIMIZATION METHODS FOR COMPLETE OBSERVABILITY

\begin{tabular}{|c|c|c|c|c|}
\hline Methods & $\begin{array}{c}\text { 14 bus } \\
\text { system }\end{array}$ & $\begin{array}{c}\text { 24 bus } \\
\text { system }\end{array}$ & $\begin{array}{c}\text { 30bus } \\
\text { system }\end{array}$ & $\begin{array}{c}\text { 57bus } \\
\text { system }\end{array}$ \\
\hline $\begin{array}{c}\text { Generalized ILP } \\
\text { Programming[14] }\end{array}$ & 4 & & 10 & 17 \\
\hline WLS[15] & $4^{\#}$ & & $10^{\#}$ & $17^{\#}$ \\
\hline Integer quadratic [17] & 4 & & 10 & 17 \\
\hline BILP [18][30] & 4 & & 10 & 17 \\
\hline CRO[28] & 4 & - & 10 & 17 \\
\hline BPSO[29] & 4 & - & 10 & 17 \\
\hline BGO[31] & 4 & - & 10 & - \\
\hline $\begin{array}{c}\text { Proposed sensitive } \\
\text { constrained ILP }\end{array}$ & $4^{*}$ & 7 & $9^{*}$ & $17^{*}$ \\
\hline
\end{tabular}

\# Optimal number of PMUs obtained solely with PMU measurements. 


\section{B. State Estimation with Sensitive Constrained PMU Allocations}

In state estimation process, load flow solution is considered as a true case for generating measurements with errors. Measurement noise (Gaussian random variable) has been added to measurement function to limit the noisy measurements. To obtain measurement accuracy for each measurement, the error variance of the measurements is added to each type of the measurement. The Gaussian measurement noise is set to $10^{-4}$. In this proposed state estimation method, voltage phasors are measured in polar coordinates and current flow measurements are measured in rectangular coordinates. As current flow measurements in polar coordinates lead to very large and uncertain for a certain range of terminal voltages and phase angles that result in an ill condition of the state estimation gain matrix [21]. Hence current measurements are considered in rectangular coordinates in the following test cases.

1) Test case 1: 14-bus system

To define the accuracy of estimation with PMU allocation at sensitive buses and to show the difference between with and without sensitive bus locations, state estimation method integrating PMU measurements without sensitive buses is shown. Three scenarios i.e., Traditional state estimation without PMU allocations, state estimation integrating PMU measurements and state estimation with proposed sensitive constrained PMU allocations are compared. In the state estimation method without considering sensitive buses, four PMUs are allocated at 2, 6, 7 and 9 to obtain 38 measurements. Total $85(38+47)$ measurements are used in state estimation method without considering sensitive buses. In this 14-bus system shown in Figure 4, four PMUs are allocated at bus 2, 6 , 9 and 14 to obtain 36 measurements and 47 conventional measurements to make the system completely observable. The traditional state estimation method is performed with 47 conventional measurements and state estimation method with proposed sensitive constrained PMU locations utilizes a total of $83(36+47)$ measurements to get the accurate performance of the state estimation.

\section{(i) Conventional measurements:}

Power injections: $\{1,2,3,4,7,8,10,11,12,14\}$

Power flows: $\{1-2,3-2,3-4,4-2,4-7,4-9,5-2,5-4,5-6,6-$ $13,7-9,11-6,12,-13\}$ buses:

(iii) PMU measurements without considering sensitive

Voltage phasors: $\{2,6,7,9\}$

Current flows: $\{2-1,2-3,2-4,2-5,6-5,6-11,6-12,6-13,7-$ 4, 7-8, 7-9, 9-4, 9-7, 9-10,9-14\}

(iii) PMU measurements considering sensitive buses:

Voltage phasors: $\{2,6,9,14\}$

Current flows: $\{2-1,2-3,2-4,2-5,6-5,6-11,6-12,6-13,9-$ 4, 9-7, 9-10, 9-14, 14-9, 14-13\}

State estimation accuracy of the system depends on variance of the estimated states. The smaller variance value implies better accuracy. For the proposed state estimation, variances of power injections and power flows are set to 0.0001, and 0.00064. For PMU measurements, variance for voltage phasors is set to 0.00001 and for real and imaginary current flows it is set to 0.001 . The state estimation solution with optimal PMU allocations converged after 6 iterations. Voltage magnitude error and phase angle error are obtained from the difference of estimated and true values (load flow calculated values are considered as true values). The voltage magnitude error and phase error obtained using traditional state estimation method without PMUs (scenario 1) and proposed state estimation with PMU (scenario2) and state estimation with proposed sensitive constrained PMU locations (scenario 3) is presented in Figure 6 and 7 respectively. Allocation of PMUs at sensitive buses has impact on measurement accuracy with complete observability of the system. That can be observed with voltage and phase angle errors obtained and presented in scenario 3. State estimation with PMU allocation at sensitive constrained buses i.e. at 2, 6, 9, and 14 shows very small angle error deviation when compared to traditional state estimation method and state estimation without sensitive constrained locations as shown in Figure 7. Comparison of scenarios 1, 2 and 3 presented in Figures 6 and 7 gives better accuracy results for complete observability of the system with the proposed sensitive constrained state estimation.

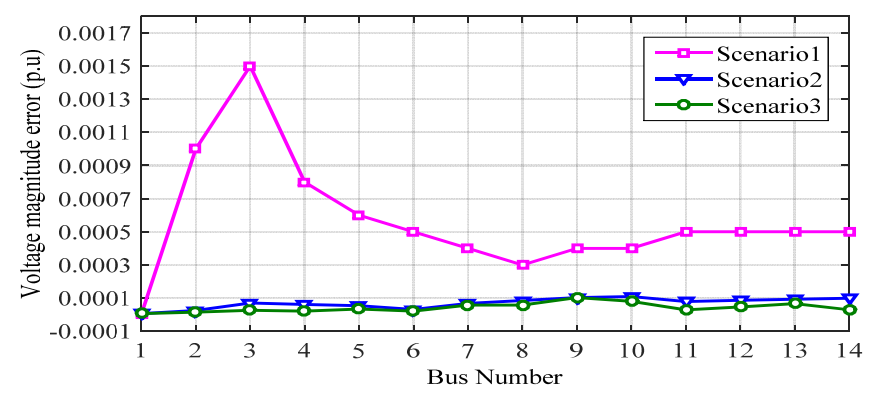

Fig. 6. Comparison voltage magnitude error of 14-Bus system

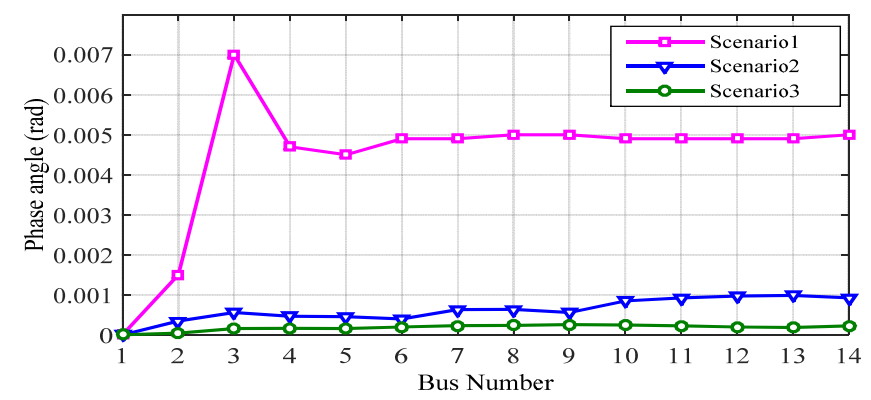

Fig. 7. Comparison of Phase angle error of 14-bus system

2) Test case 2: 57-bus system

The topology of the 57-bus system with PMUs is shown in Figure 5. The optimal PMUs obtained are 17 and are located at $\{3,4,9,12,15,20,24,25,29,31,32,33,36,38,50,54,56\}$ with sensitive constrained ILP method to make system completely observable. There are a total of 145 conventional measurements (voltage, power flow, and power injection 
measurements) and with 17 PMUs allocated in the network, 140 measurements (Voltage phasor measurements and real and imaginary current flow measurements) are obtained to get the accurate performance of the state estimation. PMU locations obtained through ILP method with and without sensitive constrained locations are shown as follows.

(i) Conventional measurements:

Power injections: $\{1,2,3,4,5,6,7,8,9,14,15,16,20,25$, $32,33,37,40,50,53,56\}$

Power flow measurements: $\{2-1,2-3,3-4,3-15,4-5,4-18$, 5-6, 6-7,6-9, 7-8, 7-29, 9-10,10-12,10-51,11-13,11-41,11-43, $12-17,13-49,14-46,15-45,18-19,19-20,21-22,22-23,23-24$, 24-25, 24-26, 27-28, 28-29, 29-52, 30-31, 32-33, 34-35,35-36, 36-40, 37-38, 37-39, 38-48, 39-57, 41-42, 41-43, 44-45, 46-47, $47-48,48-49,50-51,52-53,54-55,57-56\}$

(ii) PMU measurements obtained through general ILP Method

Voltage phasors: $\{1,4,6,13,19,22,25,27,29,32,36,39$, $41,45,47,51,54\}$

Current flows: $\{1-2,1-15,1-16,1-17,4-3,4-5,4-6,4-18,6-$ $4,6-5,6-7,6-8, \quad 13-9,13-12,13-14,13-15,13-49,19-18,19-$ $20,22-21,22-23,22-38,25-24,25-30,27-26,27-28,29-28,29-$ $52,32-31,32-33,32-34,36-35,36-37,36-40,39-37,39-57,41-$ $10,41-11,41-42,41-43,41-56,45-15,45-44,47-48,47-46,47-$ $49,51-10,51-50,54-53,54-55\}$

(iii) PMU measurements obtained through sensitive constrained ILP method

Voltage phasors: $\{3,4,9,12,15,20,24,25,29,31,32,33$, $36,38,50,54,56\}$

Current flow measurements: $\{3-2,3-15,4-3,4-5,4-6,4-18$, 9-10, 9-11, 9-12, 9-13, 9-55, 12-9, 12-13, 12-16, 12-17, 15-3, $15-1,15-13,15-14,15-45,20-19,20-21,24-23,24-26,25-24$, $25-30,29-7,29-28,29-52,31-32,31-30,32-33,32-34,33-$ $32,33-34,36-35,36-37,36-40,38-22,38-37,38-44,38-48,38-$ $49,50-49,50-51,54-55,54-53,56-57,56-41,56-42,56-40\}$

The PMU measurements obtained through general ILP method are 140 and with including conventional measurements, total measurements are $285(145+140)$. With the integration of PMU measurements obtained through sensitive constrained ILP and conventional measurements, we obtain total 278 $(145+133)$ measurements to perform state estimation. For the proposed method, variances of power injections, power flows and voltage phasors of PMU measurements are set to 0.00001 and for real and imaginary current flow measurements it is set to 0.001 . The state estimation solution with optimal PMU allocations converged after 7 iterations. The voltage magnitude error and phase error obtained for 57 bus system using traditional state estimation method without PMUs (scenario 1), state estimation with PMU (scenario 2) and state estimation with proposed sensitive constrained PMU allocation (scenario 3 ) is presented in Figures 8 and 9 respectively. With PMUs allocation at sensitive buses, state error obtained in the measurement is very low which means improved accuracy of the state estimation.

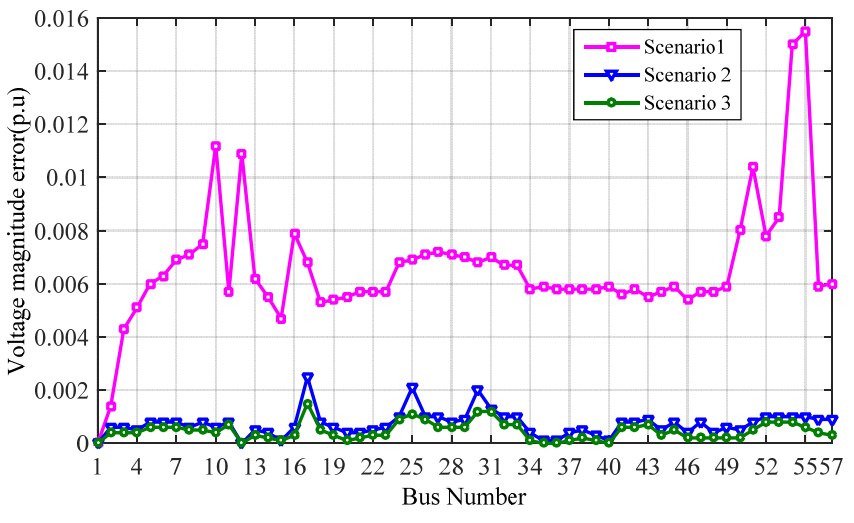

Fig. 8. Comparison voltage magnitude error of 57-Bus system

Phase angle measurement is very important in power system state estimation, as a small deviation of angle leads to instability of the system. Comparison of scenario 1 (Traditional state estimation without PMU), scenario 2 (State estimation with PMU) and scenario 3 (State estimation with proposed sensitive bus constrained PMU allocation) in Figure 9 shows the state estimation method with sensitive constrained PMU allocation (Scenario 3) results in very small phase angle error deviation showing the impact of PMU measurement accuracy with complete observability of the system.

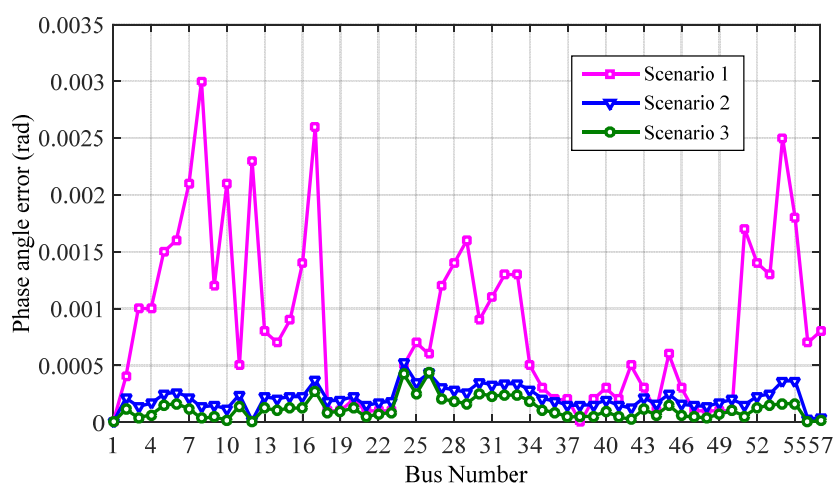

Fig. 9. Comparison of Phase angle error of 57-bus system

C. Performance of State Estimation with Proposed Sensitive Constrained Allocation with Root Mean Square Error (RMSE) Indicator

In order to quantify accuracy and performance of state estimation, an RMSE is formulated and error is computed. The states of 14- and 57-bus systems with optimal sensitive constrained PMUs allocation are examined with RMSE and compared with traditional state estimation and state estimation method without considering sensitive buses. RMSE is formulated as

$$
\mathrm{RMSE}=\sqrt{\frac{1}{n} \sum_{k=1}^{n}\left(x_{\text {est }}(k)-x_{t r u}(k)\right)^{2}}
$$


where $x_{\text {est }}$ is estimated state, $x_{\text {tru }}$ is the true state of the system, and $\mathrm{n}$ is the number of buses in the system. RMSE for voltage and phase angles are computed separately because the scale of measurements is different in each case. RMSE measures the accuracy of state estimation voltage and phase angle errors of the system. RMSE with PMU for the proposed sensitive approach has better accuracy compared to traditional state estimation and all other methods. RMSE values of 14- and 57bus systems are shown in Table X. When compared to state estimation solution given in $[19,21,26,27]$, the solution with proposed method is better.

TABLE X. COMPARISON OF RMSE INDEX OF PROPOSED METHOD WITH OTHER METHODS

\begin{tabular}{|c|c|c|}
\hline \multirow{2}{*}{ Methods } & \multicolumn{2}{|c|}{ RMSE of Voltage (p.u) } \\
\cline { 2 - 3 } & $\begin{array}{c}\text { IEEE 14-Bus } \\
\text { system }\end{array}$ & $\begin{array}{c}\text { IEEE57- } \\
\text { Bus System }\end{array}$ \\
\hline $\begin{array}{c}\text { Traditional State Estimation } \\
\text { Method[22] }\end{array}$ & 0.0006 & 0.007 \\
\hline Ref[19] & 0.000076 & 0.002 \\
\hline Ref[21] & 0.00011 & - \\
\hline Ref[27] & 0.00099 & - \\
\hline $\begin{array}{c}\text { Proposed sensitive constrained } \\
\text { state estimation method }\end{array}$ & 0.000027 & 0.0009 \\
\hline
\end{tabular}

\section{CONCLUSION}

This paper presented a sensitive constrained integer linear programming approach for optimal allocation of PMUs considering sensitive buses giving the highest priority in the system for state estimation solution. This approach for optimal PMU allocation with zero injection modeling is able to minimize the number of PMUs in case of single and no line contingency without losing complete observability of the system. With the optimal allocation of PMUs at sensitive buses, redundancy of the system is improved and resulted in economic benefit. Allocation of PMUs at sensitive buses enhanced the accuracy and performance of the state estimation solution. Results obtained from the simulations of 14, 30 and 57 bus systems show the effectiveness of the proposed method when compared with other methods.

\section{REFERENCES}

[1] K.. E. Martin, D. Hamai, M. G. Adamiak, S. Anderson, M. Begovic, G. Benmouyal, G. Brunello, J. Burger, J. Y. Cai, B. Dickerson, V. Gharpure, B. Kennedy, D. Karlsson, A. G. Phadke, J. Salj, V. Skendzic, J. Sperr, Y. Song, C. Huntley, B. Kasztenny, E. Price, "Exploring the IEEE Standard C37.118-2005 synchrophasors for power systems", IEEE Transactions on Power Delivery, Vol. 23, No. 4, pp. 1805-1811, 2008

[2] L. L. Lai, H. T. Zhang, S. Mishra, D. Ramasubramanian, C. S. Lai, F. Y. Xu, "Lessons Learned from July 2012 Indian Blackout", $9^{\text {th }}$ IET International Conference on Advances in Power System Control, Operation and Management, pp. 1-6, 2012

[3] T. Moger, T. Dhadbanjan, "A novel index for identification of weak nodes for reactive compensation to improve voltage stability IET Generation, Transmission \& Distribution, Vol. 9, No. 14, pp. 1826-1834, 2015

[4] F. Capitanescu, T. V. Cutsem, "Unified sensitivity analysis of unstable or low voltages caused by load increases or contingencies", IEEE Transactions on Power Systems, Vol. 20, No. 1, pp. 321-329, 2005.

[5] V. Balamourougan, T. S. Sidhu, M. S. Sachdev, "Technique for online prediction of voltage collapse IEE Proceedings - Generation, Transmission and Distribution, Vol. 151, No. 4, pp. 453-460, 2004
[6] I. Musirin, T. K. A. Rahman, "On-line Voltage stability based contingency ranking using fast voltage stability index (FVSI)", Transmission and Distribution Conference and Exhibition 2002: Asia Pacific. IEEE/PES,Vol. 2, pp. 1118-1123, 2002

[7] B. Divya, R. Devarapalli, "Estimation of sensitive node for IEEE-30 bus system by load variation", Green Computing Communication and Electrical Engineering, pp. 1-4, 2014

[8] A. G. Phadke, J. S. Thorp, K. J. Karimi, "State estimation with phasor measurements", IEEE Power Engineering Review, Vol. PER-6, No. 2, pp. 48,1986

[9] A.i Abur, F. H. Magnago, "Optimal meter placement for maintaining observability during single branch outages", IEEE Transactions on Power Systems, Vol. 14, No. 4, pp. 1273-1278, 1999

[10] F. Aminifar, C. Lucas, A. Khodaei, M. Fotuhi-Firuzabad, "Optimal placement of phasor measurement units using immunity genetic algorithm", IEEE Transactions on Power Delivery, Vol. 24, No. 3, pp. 1014-1020, 2009

[11] B. Milosevic, M. Begovic, "Nondominated sorting genetic algorithm for optimal phasor measurement placement", IEEE Transactions on Power Systems, Vol. 18, No.1, pp. 69-75, 2003

[12] K. G. Khajeh, E. Bashar, A. M. Rad, G. B. Gharehpetian, "Integrated model considering effects of zero Injection buses and conventional measurements on optimal PMU placement", IEEE Transactions on Smart Grid, Vol. 8 , No. 2, pp. 1006-1013, 2017

[13] D. Dua, S. Dambhare, R. K. Gajbhiye, S. A. Soman, "Optimal Multistage Scheduling of PMU Placement: An ILP Approach", IEEE transactions on Power Delivery, Vol. 23, No. 4, pp. 1812-1820, 2008

[14] B. Gou, "Generalized Integer Linear Programming formulation for optimal PMU placement", IEEE Transactions on Power Systems, Vol. 23, No. 3, pp. 1099-1104, Aug. 2008

[15] N. M. Manousakis, G. N. Korres, "A weighted least squares algorithm for optimal PMU placement", IEEE Transactions on Power Systems, Vol. 28 , No. 3 pp. 3499-3500, 2013

[16] S. Chakrabarti, E. Kyriakides, "Optimal placement of phasor measurement units for power system observability", IEEE Transactions on Power Systems, Vol. 23, No. 3, pp. 1433-1440, 2008

[17] S. Chakrabarti, E. Kyriakides, D. G. Eliades, "Placement of synchronized measurements for power system observability", IEEE Transactions on Power Systems, Vol. 24, No. 1, pp. 12-19, 2009

[18] N. H. Abbasy, H. M Ismail, "A unified approach for the optimal PMU location for power system state estimation", IEEE Transactions on Power Systems, Vol. 24, No. 2, pp. 806-813, 2009

[19] G. Valverde, S. Chakrabarti, E. Kyriakides, V. Terzija, "A constrained formulation of hybrid state estimation", IEEE Transactions on Power Systems, Vol. 26, No. 3, pp. 1102-1109, 2011

[20] A. Simoes Costa, A. Albuquerque, D. Bez, "An estimation fusion method for including phasor measurements in to power system", IEEE Transactions on Power Systems, Vol. 28, No. 2, pp. 1910-1920, 2013

[21] S. Chakrabarti, E. Kyriakides, G. Ledwich, A. Ghosh, "Inclusion of PMU current phasor measurements in a power system state estimator", IET Generation, Transmission \& Distribution, Vol. 4, No. 10, pp. 11041115,2009

[22] A. Abur, A. G. Exposito, Power system sate estimation: Theory and Implementations, Taylor \& Francis, 2004

[23] A. G. Padke, J. S. Thorp, "Synchrophasor Measurements and their Applications", Power electronics and power systems, Springer, 2008

[24] B. Xu, A. Abur, "Observability analysis and measurement placement for systems with PMUs", IEEE PES. Power systems conference and Exposition, Vol. 2, pp. 943-946, 2004

[25] C. Bruno, C. Candia, L. Franchi, G. Giannuzzi, M. Pozzi, R. Zaottini, M. Zaramella, "Possibility of enhancing classical weighted least squares state estimation with linear PMU measurements", IEEE Bucharest Power Tech, pp. 2-7, 2009

[26] R. F. Nuqui, A. G. Phadke, "Hybrid linear state estimation utilizing synchronized phasor measurements", IEEE Lausanne Power Tech, pp. $1665-1669,2007$ 
[27] G. N. Korres, N. M. Manousakis, "State estimation and observability analysis for phasor measurement unit measured systems", IET Generation, Transmission \& Distribution, Vol. 6, No. 9, pp. 902-913, 2012

[28] J. Xu, M. H. F. Wen, V. O. K. Li, K. C. Leung, "Optimal PMU placement for wide-area monitoring using chemical reaction optimization", IEEE PES Innovative Smart Grid Technologies Conference, pp. 1-6, 2013

[29] A. Ahmadi, Y. Alinejad-Beromi, M. Moradi, "Optimal PMU placement for power system observability using binary particle swarm optimization and considering measurement redundancy", Expert Systems with Applications, Vol. 38, No. 6, pp. 7263-7269, 2011

[30] M. Ravindra, R. Srinivasa Rao. "Dynamic state estimation solution with optimal allocation of PMUs in presence of load changes", IEEE International Conference on Intelligent Control Power and Instrumentation, pp. 163-168, 2016

[31] K. Jamuna, K. S. Swarup, "Multi-objective biogeography based optimization for optimal PMU placement", Applied Soft Computing, Vol. 12, No.5, pp. 1503-1510, 2012 